\title{
Le diagnostic génétique pré-implantatoire : premier bilan du groupe parisien
}

\author{
Nelly ACHOUR-FRYDMAN2, Gérard TACHDJIAN2, Pierre RAY3, Serge ROMANA3, \\ Samir HAMAMAH2, Sabine MARCADET-FREDET1, Violaine KERBRAT1, Renato FANCHIN1, \\ Jacques KADOCH1, Tania ATTIE3, M. LELORC'H3, Michel VEKEMANS3, Arnold MUNNICH3, \\ René FRYDMAN1
}

\begin{abstract}
1Service de Gynécologie-Obstétrique, Clamart 2Service d'Histologie-Embryologie-Cytogénétique à orientation Biologique et Génétique de la Reproduction, Hôpital Antoine Béclère (AP-HP), Clamart

3Département de Génétique, Hôpital Necker-Enfants malades, Paris
\end{abstract}

\section{RESUME}

Le travail rapporte l'expérience parisienne du diagnostic génétique pré-implantatoire (DPI). 59 couples ont été pris en charge de janvier 2000 à juillet 2001 au cours de 71 cycles. Les ovocytes recueillis ont été inséminés par injection intracytoplasmique de spermatozoïde et les embryons biopsiés au troisième jour de développement. L'analyse génétique a été effectuée le jour de la biopsie et la majorité des transferts embryonnaires effectués le quatrième jour post fécondation. Au cours des 71 cycles, 872 ovocytes ont été recueillis dont 731 étaient compatibles avec une injection intracytoplasmique de spermatozoïde. Parmi les 505 embryons obtenus, 421 embryons ont été biopsiés et un résultat génétique a été obtenu pour $312(74 \%)$ d'entre eux. 127 embryons ont été transférés au cours de 58 transferts. 18 grossesses biologiques et 12 cliniques ont été obtenues ( 7 uniques, 4 gémellaires et une triple). 16 enfants sont nés. Le DPI prend désormais sa place parmi les choix proposés aux couples à risque de transmission de maladie génétique de particulière gravité et incurable au moment du diagnostic.

Mots-clés : diagnostic génétique pré-implantatoire, maladie chromosomique, maladie monogénique.

\section{INTRODUCTION}

Le diagnostic génétique pré-implantatoire (DPI) est une alternative au diagnostic prénatal. Il consiste à réaliser une analyse génétique sur des embryons humains obtenus par fécondation in vitro et à ne procéder au transfert intrautérin que des embryons sains ou porteurs sains.

Le DPI a été possible grâce au développement des techniques de biologie moléculaire permettant une analyse génétique sur cellule unique, principalement la réaction en chaîne de la polymérase (PCR) et l'hybridation in situ avec des sondes fluorescentes (FISH).

Si l'annonce de la première grossesse obtenue après DPI fut relatée en 1990 en Angleterre [9], en France ce n'est qu'en été 1999 que l'Assistance Publique - Hôpitaux de Paris (AP-HP) (Hôpital Necker et A. Béclère) et le Centre Hospitalo-Universitaire de Schiltigheim, bientôt rejoints par l'hôpital A. de Villeneuve de Montpellier, ont été autorisés à pratiquer le DPI.

Le législateur a encadré la pratique du DPI. Cet examen s'adresse aux seuls couples qui ont une forte probabilité de donner naissance à un enfant atteint d'une maladie génétique d'une particulière gravité reconnue comme incurable au moment du diagnostic.

\section{Correspondance :}

Pr René Frydman - Service de Gynécologie-Obstétrique, Hôpital Antoine Béclère (AP-HP), 157 Rue de la Porte de

Trivaux, 92141 Clamart. - Tel 33.1.46.31.38.30 -

Email secret.pr-frydman@abc.ap-hop-paris.fr 
Nous rapportons ici l'expérience du groupe parisien constitué des services de Gynécologie-Obstétrique, et de Biologie de la Reproduction (A. Béclère) et du Département de Génétique (Necker) réunis au sein d'une Fédération de l'Assistance Publique - Hôpitaux de Paris (AP-HP).

\section{PATIENTS ET METHODES}

\section{Recrutement des couples}

De Janvier 1999 à Juillet 2001, nous avons reçu en consultation 230 couples.

Parmi les 230 couples venus de toutes les régions de France, 136 demandes ont été retenues après accord de l'une des deux Commissions pluridisciplinaires du diagnostic prénatal de la FAMA Necker-Béclère dont l'avis est statutairement requis selon la législation en vigueur.

Parmi les 94 couples récusés, 14 l'ont été en raison de non éligibilité de l'indication de DPI et 80 en raison de la non faisabilité du diagnostic sur cellule unique.

Au cours de cette consultation, les taux de succès, les aléas éventuels de la fécondation in vitro (FIV) ainsi que du DPI ont été abordés ainsi que l'éventualité du recours au diagnostic anténatal en cas de grossesse.

En cas d'acceptation de l'indication, un bilan pré-fécondation in vitro (FIV) a été prescrit.

Un entretien psychologique a été systématiquement évoqué au cours de la première consultation, 86 couples y ont eu recours.

Au total, parmi les 136 couples qui ont été acceptés par l'équipe médicale, 59 ont bénéficié de janvier 2000 à juillet 2001 de 97 cycles de FIV avec DPI ( 26 cycles ont été annulés soit $26,8 \%$ des cycles débutés). Soixante et onze tentatives avec ponctions ovocytaires et biopsie embryonnaire ont été réalisés dans les services de Gynécologie Obstétrique et de Biologie de la Reproduction de l'hôpital Antoine Béclère. A ces 59 couples, il faut ajouter 3 couples ayant des embryons congelés provenant d'autres centres de FIV qui ont bénéficié à Antoine Béclère d'une biopsie embryonnaire pour DPI sur les embryons congelés. L'analyse génétique du ou des blastomères a été effectuée dans le département de génétique de l'hôpital Necker.

\section{Caractéristiques des couples}

Nous distinguerons les maladies chromosomiques et les maladies monogéniques car les populations concernées et les techniques proposées ne sont pas identiques.

La répartition des affections pour lesquelles un DPI a été réalisé est indiquée dans les Tableaux 1 et 2 . Vingt-cinq couples (groupe 1) présentaient un risque de déséquilibre chromosomique (Tableau 1) et 34 couples (groupe 2) pré-
Tableau 1 : Désordres chromosomiques ayant fait l'objet d'une stimulation et d'une ponction en vue d'un DPI (groupe 1).

\begin{tabular}{|c|c|c|c|}
\hline & \multicolumn{3}{|c|}{ Couples } \\
\hline & $\begin{array}{l}\text { Femmes } \\
\text { porteuses }\end{array}$ & $\begin{array}{l}\text { Hommes } \\
\text { porteurs }\end{array}$ & Total \\
\hline \multicolumn{4}{|l|}{ Anomalies de structure } \\
\hline $\begin{array}{l}\text { Translocations Robertsor } \\
\operatorname{der}(13 ; 14) \text { (q10;q10) } \\
\operatorname{der}(13 ; 22) \text { (q10;q10) } \\
\operatorname{der}(14 ; 21)(\mathrm{q} 10 ; q 10)\end{array}$ & $\begin{array}{r}\text { liennes : } \\
4 \\
1 \\
1\end{array}$ & $\begin{array}{l}7 \\
0 \\
4\end{array}$ & $\begin{array}{c}11 \\
1 \\
5\end{array}$ \\
\hline \multicolumn{4}{|c|}{ Translocations Réciproques: } \\
\hline $\mathrm{t}(13 ; 14)(\mathrm{q} 34 ; \mathrm{q} 13)$ & 1 & 0 & 1 \\
\hline $\mathrm{t}(2 ; 22)(\mathrm{q} 24 ; \mathrm{q} 11)$ & 0 & 1 & 1 \\
\hline $\mathrm{t}(8 ; 14)(\mathrm{q} 22 ; \mathrm{q} 23)$ & 0 & 1 & 1 \\
\hline $\mathrm{t}(11 ; 22)(\mathrm{q} 25 ; \mathrm{q} 12)$ & 1 & 0 & 1 \\
\hline $\mathrm{t}(14 ; 18)(\mathrm{q} 21 ; \mathrm{q} 12)$ & 0 & 1 & 1 \\
\hline $\mathfrak{t}(18 ; 21)(\mathrm{q} 21 ; \mathrm{q} 22)$ & 0 & 1 & 1 \\
\hline$t(5 ; 6)(q 36 ; q 24)$ & 1 & 0 & 1 \\
\hline $\begin{array}{l}\text { Anomalies de nombre } \\
\text { Trisomie } 8 \text { en } \\
\text { mosaique }(50 \%)\end{array}$ & 0 & 1 & 1 \\
\hline Total & 9 & 16 & 25 \\
\hline
\end{tabular}

sentaient un risque de transmission d'une maladie monogénique (Tableau 2). Concernant le groupe 1, les porteurs de ces translocations sont répartis en $64 \%$ d'hommes et $36 \%$ de femmes.

$\mathrm{Au}$ sein du groupe 2, il existe une répartition équivalente des maladies géniques autosomiques ou liées à l'X. Parmi les maladies autosomiques, on note la place prépondérante de la mucoviscidose.

Les principaux antécédents obstétricaux sont caractérisés pour le groupe 1 par la présence d'une infertilité primaire ou secondaire (associée et d'une fréquence élevée de fausses couches spontanées) : 42 FCS pour 25 couples versus 12 pour 34 couples dans le groupe 2 . A l'inverse, le nombre d'interruptions médicales de grossesse (IMG) est beaucoup plus élevé dans le groupe 2 ( 24 sur 27 IMG totalisées dans les deux groupes).

\section{Diagnostic génétique pré-implantatoire}

Les ovocytes recueillis ont été inséminés par injection intra-cytoplasmique de spermatozoïde (ICSI). Au troisième jour de développement la biopsie embryonnaire a été réalisée, sous microscope inversé équipé de micromanipulateurs. A ce stade les embryons possèdent entre 6 et 8 cellules. Un orifice a été pratiqué dans la zone pellucide à l'aide d'un faisceau laser (Laser Fertilase, Société MTG, Altdorf, Allemagne). Une ou deux cellules ont ensuite été prélevées 
Tableau 2 : Affections géniques ayant fait l'objet d'une stimulation et d'une ponction en vue d'un DPI (groupe 2).

\section{Couples}

\section{Autosomiques récessives}

Amyotrophie spinale

Mucoviscidose

5

9*

Autosomiques dominantes

Myopathie de Steinert

Liées à l'X

Retard mental lié à l'X

Myopathie de Duchenne

Déficit en Ornithine Carbamyl Transferase 1

Dysplasie spondylo-épiphysaire tardive 1

Syndrome de Vactrel

Maladie de Pelizaeus Merzbacher

Myopathie de Becker 1

Maladie de Menkes 1

Hémophilie A

Pathologie mixte

Mucoviscidose et retard mental lié à l'X

Total

1

34

(*) 4 patients étaient porteurs d'une agénésie bilatérale des canaux déférents

par aspiration dans une micropipette de $35 \mathrm{~mm}$ de diamètre interne. L'embryon a été alors replacé dans un milieu de culture jusqu'au moment du transfert [17].

Dans le cas où le diagnostic utilisait l'amplification d'une séquence d'ADN par réaction en chaîne PCR (Polymerase Chain Reaction), la cellule a été placée dans un tube contenant un tampon de lyse permettant d'extraire l'ADN du noyau sous contrôle visuel sous loupe binoculaire. En revanche, lorsque le diagnostic utilisait l'hybridation in situ avec sonde d'ADN fluorescente ou FISH, la cellule a été placée sur une lame de verre contenant de l'acide chlorhydrique permettant de libérer le noyau à analyser [7, 8] (groupe 1).

La technique de FISH est utilisée pour les indications chromosomiques. Les sondes d'ADN utilisées sont spécifiques de la région du chromosome impliqué dans un remaniement de structure $[10,15]$ ou spécifiques des chromosomes $\mathrm{X}$ et $\mathrm{Y}$ lorsqu'il s'agit d'un diagnostic de sexe et dans certaines maladies liées à l'X.

Pour les autres affections géniques, la méthode utilisée est la PCR [11].

\section{Transfert embryonnaire}

Le résultat de l'analyse génétique a été rendu le soir même ou le lendemain de la biopsie, les transferts embryonnaires ont eu lieu dans la majorité des cas le quatrième jour de développement.
Les embryons sains ou porteurs sains surnuméraires ont été laissés en culture jusqu'au sixième jour et congelés s'ils avaient atteint le stade de blastocyste. Pour trois couples le DPI a été réalisé à partir d'embryons congelés obtenus dans d'autres centres au cours de FIV précédentes lorsque le DPI n'était pas encore autorisé.

\section{RESULTATS}

Les patientes étaient âgées de 32,7 ans en moyenne (24 à 40 ans).

De Janvier 2000 à Juillet 2001, 71 cycles ayant abouti à une ponction sont analysés. Une moyenne de 12,3 ovocytes a été recueillie dont $84 \%$ ont pu être inséminés. 421 embryons ont pu être biopsiés. Un résultat génétique a été obtenu pour 312 d'entre eux (74\%). Parmi ceux-ci 127 ont été transférés au cours de 58 transferts (soit une moyenne de 2,2 embryons par transfert). Treize transferts n'ont pas été réalisés car l'ensemble des embryons était atteint.

Le Tableau 3 indique le nombre de grossesses évolutives par ponction et par transfert, ainsi que les naissances obtenues après DPI. Ces résultats incluent les transferts d'embryons décongelés ayant bénéficié d'un DPI.

Au total, 61 transferts ont été effectués, 20 dosages positifs de l'hCG ont été notés (33\%), parmi lesquels 7 sont restés biochimiques ( $>100$ et $<1000$ UI hCG), une fausse-couche est survenue à 10 semaines d'aménorrhée et 12 grossesses ont été évolutives $(19,7 \%)$ avec 16 enfants attendus $(26,2 \%)$ dont 14 sont déjà nés.

Parmi les 132 embryons frais et décongelés transférés, 19 activités cardiaques ont été observées soit un taux d'implantation embryonnaire de $14,4 \%$ et de $31,1 \%$ par transfert.

Concernant la fausse couche qui s'est produite à 10 semaines d'aménorrhée : il s'agit d'un DPI pratiqué pour une translocation $(13 ; 14)$ paternelle. Le produit de fausse couche a pu être récupéré, l'analyse chromosomique faite dans de bonnes conditions techniques n'a pas retrouvé d'aneuploïdie.

Parmi les 12 grossesses évolutives ayant dépassé 12 semaines d'aménorrhée, sept grossesses étaient simples, 4 grossesses étaient gémellaires mais une s'est réduite spontanément en grossesse simple et une grossesse triple a été réduite médicalement en grossesse gémellaire au terme de 11 semaines d'aménorrhée.

C'est ainsi que nous pouvons à ce jour rapporter les 16 premières naissances issues de 4 grossesses gémellaires (dont la grossesse triple réduite) et de 8 grossesses simples.

Un prélèvement de sang de cordon a été effectué systématiquement à la naissance afin de confirmer le DPI. Aucune 
Tableau 3 : Grossesses évolutives et naissance après DPI (Janvier 2000-Juillet 2001).

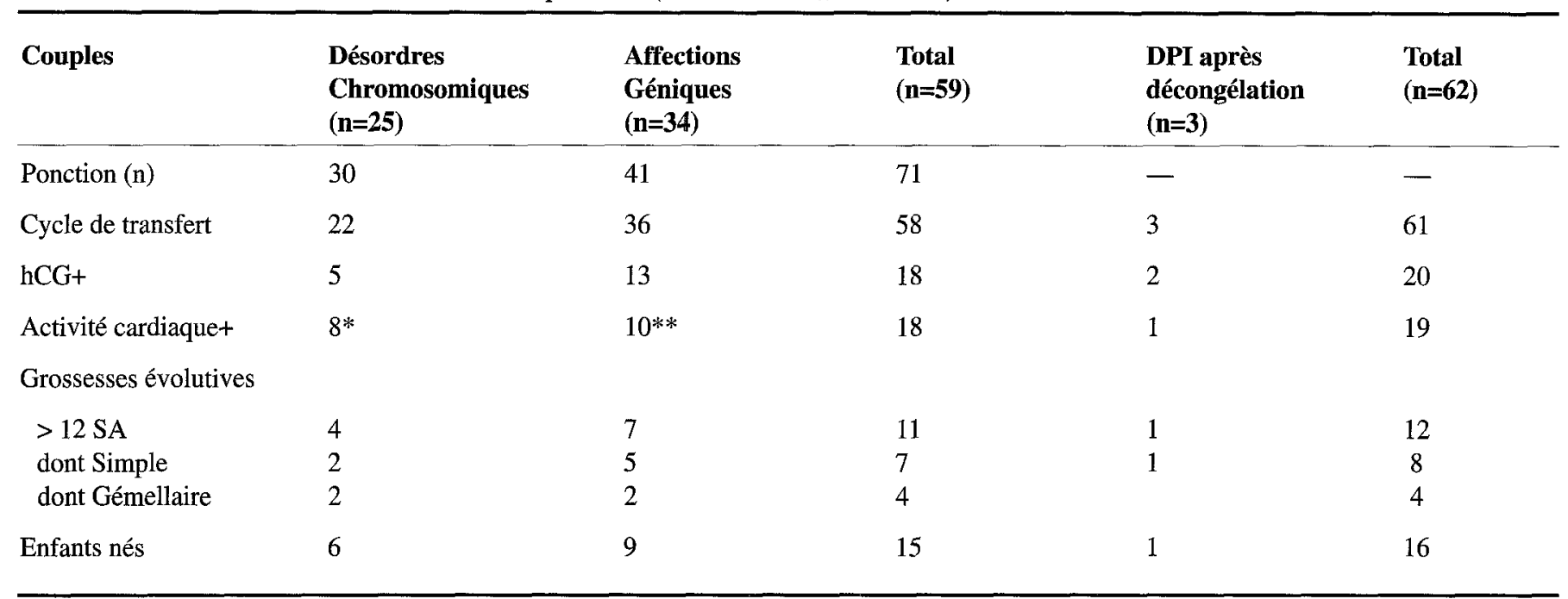

(*) Une fausse couche à $10 \mathrm{SA}$, une réduction médicale de triple à gémellaire.

(**) Une réduction embryonnaire spontanée d'une grossesse gémellaire.

erreur de diagnostic n'a été notée. Tous les enfants ont été examinés par un pédiatre à la naissance. Une étude de suivi à 1 an et 3 ans est actuellement en place.

\section{DISCUSSION}

La liste des maladies génétiques ayant pu bénéficier d'un DPI a été récemment mise à jour [3].

Compte tenu des moyens limités dont nous disposons, nous avons concentré nos efforts de mise au point sur des mutations assez fréquentes, touchant un nombre important de patients (mucoviscidose, amyotrophie spinale, maladie de Steinert). Au sein du Groupe d'Etude et de Travail du DPI en France (GET-DPI) nous avons proposé une organisation en fonction des spécificités de chaque centre avec en particulier pour le groupe parisien la mise au point du diagnostic de la drépanocytose, du déficit en Ornithine Carbamyl Transférase (qui a abouti à la première naissance d'un enfant né en France après DPI [13]) et du syndrome de l'X fragile, le groupe strasbourgeois développant également la prise en charge de l'X fragile, de la maladie de Huntington et de l'achondroplasie. Le groupe parisien a par ailleurs initié en France la prise en charge des risques chromosomiques ( 25 cas).

La population des couples porteurs d'anomalies chromosomiques se singularise par l'existence fréquente d'une infertilité qui peut être notre biais de recrutement de cette population.

En effet au cours d'un bilan d'infertilité masculine relevant de l'injection intra-cytoplasmique (ICSI), le caryotype peut révéler une anomalie chromosomique. La fréquence des remaniements chromosomiques étant de 2 à $6 \%$ dans la population infertile [12]. Cependant, comme l'ont montré certains auteurs $[5,18]$, dans un pourcentage de cas à peu près équivalent, la conjointe de l'homme infertile peut ellemême être porteuse d'un remaniement chromosomique. Ce qui rend absolument nécessaire la réalisation du caryotype du couple avant toute ICSI, afin de pouvoir bénéficier d'un conseil génétique qui peut aboutir soit à une demande de DPI soit à une prise en charge en diagnostic anténatal.

La population des couples porteurs d'un risque génique est dans notre expérience très différente. Ce ne sont pas des couples infertiles mais au contraire des couples fertiles éprouvés par la maladie de leurs enfants ( 1 ou 2$)$ à charge ou ayant subi parfois plusieurs interruptions médicales de grossesse (IMG). S'il est vrai qu'une IMG est toujours traumatisante, nous avons été frappés, et c'est sans doute le biais du recrutement de la consultation de DPI, par le vécu difficile des couples qui se tournent vers le DPI en raison d'une prise en charge jugée comme insuffisante du diagnostic anténatal.

Parmi les 215 accouchements rapportés par le Consortium Européen du DPI, le taux et le type de complications gravidiques relevés après l'analyse de blastomères $[1-3]$ apparait pour l'instant comparable aux données habituelles de la littérature de même que les naissances après analyse du 1er globule polaire [16].

Ce premier bilan montre que la pratique du DPI s'est développée relativement rapidement dans notre groupe et qu'après 18 mois de fonctionnement, nous avons pu, en bénéficiant de l'expérience des plus anciens, offrir des résultats comparables aux données du Consortium Européen qui réunit 25 centres pour l'année 2001 . 
Finalement, si le DPI a permis la naissance d'enfants indemnes de la maladie recherchée, il a, en récusant le transfert dans 13 cas où il n'y avait que des embryons atteints disponibles, permis d'éviter la survenue d'enfants atteints de maladies graves et incurables ce qui est également l'objectif poursuivi. Dans l'analyse globale du bilan du DPI, ce point (absence de transfert) mérite d'être noté comme un élément positif.

Cependant, ce bilan positif doit être tempéré par le fait qu'un grand nombre de maladies orphelines ne peuvent être prises en charge rapidement du fait du manque de moyens pour effectuer les mises au point diagnostiques préalables.

De nouvelles perspectives diagnostiques s'ouvrent, telles les maladies mitochondriales mais il faut noter l'apparition de deux indications qui existent à l'échelle européenne et ne sont pas autorisées en France : le DPI pour risque d'aneuploïdie et le DPI pour choix de sexe qui ont été mentionnés dans le dernier rapport du Consortium Européen [2].

Pour le premier cas, il s'agit soit de patients en cours de FIV âgés de plus de 38 ans $(n=101)$, soit de patients jeunes ayant eu déjà 3 échecs de FIV $(n=45)$ ou plusieurs fausses couches ( $n=116)$ à qui l'on a proposé une FIV afin de diagnostiquer les embryons aneuploïdes. Au total pour 2001, 331 cycles ont été réalisés en Europe par 11 centres pour cette indication. Cette indication vise à diminuer le taux de fausses couches spontanées [6].

78 couples ont été traités pour choix du sexe par 3 centres européens. Cette indication a soulevé de la part de notre groupe une réprobation [14] considérant que le DPI comme le diagnostic anténatal (DAN) ne doivent pas être effectués à des fins de convenances hors de tout risque de pathologie médicale.

Ce dernier point pose de façon cruciale la cohérence des politiques de santé à l'échelle européenne.

Une troisième indication mérite réflexion c'est celle d'une demande de DPI pour éviter une maladie génétique (maladie de Franconi) associée à une identification du groupe HLA en vue de pouvoir greffer des cellules souches hématopoïétiques compatibles à un aîné (sans donneur compatible).

Le Comité National d'Ethique a été saisi de ces demandes aujourd'hui récusées en France et n'a pas encore donné de réponse.

Enfin, il ne faut pas perdre de vue la difficulté du diagnostic sur une cellule et le Consortium Européen relève 8 erreurs de diagnostic dont 2 en post-natal. Afin de diminuer le risque d'erreur, de nouvelles attitudes peuvent être préconisées : biopsie sur deux cellules prélevées plutôt qu'une, et surtout développement de nouvelles techniques telles que la PCR multiplex, la PCR fluorescente [4], linkage analysis ou encore le développement de l'hybridation génomique comparative (CGH) [19].

Le DPI prend place dans les possibilités offertes aux couples à risque de transmission d'une maladie génétique. La lourdeur de la procédure, les incertitudes du résultat doivent toujours être mises en balance avec les alternatives en particulier un diagnostic prénatal bien conduit.

Il n'en reste pas moins qu'une page est tournée, l'embryon humain « malade » d'invisible et d'intouchable qu'il était devient sujet d'étude et peut être demain deviendra un sujet qui bénéficiera d'un traitement.

\section{Remerciements :}

Ils vont à l'ensemble des médecins, ingénieurs, sages-femmes, techniciennes, infirmières, secrétaires, qui constituent les équipes de la F.A.M.A Necker-Béclère. Ils ont par leurs activités permis la réalisation $d u$ DPI.

\section{REFERENCES}

1. ESHRE PREIMPLANTATION GENETIC DIAGNOSIS (PGD) CONSORTIUM : Preliminary assessment of data from January 1997 to September 1998. ESHRE PGD Consortium Steering Committee. Hum. Reprod., 1999, 14 : 3138-3148.

2. ESHRE PREIMPLANTATION GENETIC DIAGNOSIS (PGD) CONSORTIUM : Data collection II (May 2000). Hum. Reprod., $2000,15: 2673-2683$.

3. ESHRE PREIMPLANTATION GENETIC DIAGNOSIS (PGD) CONSORTIUM : Data collection III. Hum. Reprod., 2002, 1 : 233-246.

4. FINDLAY I., MATTHEWS P.L., MULCAHY B.K., MITCHELSON K. : PCR to diagnose multiple defects from single cells : implications for PGD. Mol. Cell. Endocrinol., 2001, 183 suppl 1 : S5-S12.

5. GEKAS J., THEPOT F., TURLEAU C. et al. : Chromosomal factors of infertility in candidate couples for ICSI : an equal risk of constitutional aberrations in women and men. Hum. Reprod., 2001, $16: 82-90$.

6. GIANAROLLI L., MAGLI M.C., FERRARETTI A.P. : The in vivo and in vitro efficiency and efficacy of PGD for aneuploidy. Mol. Cell. Endocrinol., 2001, 183 supl 1 : S13-S18.

7. GRIFFIN D.K., HANDYSIDE A.H., PENKETH R.J., WINSTON R.M., DELHANTY J.D. : Fluorescent in situ hybridation to interphase nuclei of human preimplantation embryos with $\mathrm{X}$ and Y chromosome specific probes. Hum. Reprod., 1991, 6 : 101-105.

8. GRIFFIN D.K, HANDYSIDE A.H., HARPERT J.C. et al. : Clinical experience with preimplantation diagnosis of sex by dual fluorescent in situ hybridization. J. Assist. Reprod. Genet., 1994, $11: 132-143$.

9. HANDYSIDE A., LESKO J., TARIN J., WINSTON R., HUGUES M. : Birth of a normal girl after in vitro fertilization and preimplantation diagnostic testing for cystic fibrosis. N. Engl. J. Med., 1992, 327 : 905-909. 
10. KNIGHT S.J., LESE C.M., PRECHT K.S. et al. : An optimized set of human telomere clones for studying telomere integrity and architecture. Am. J. Hum. Genet., 2000, 67 : 320-332.

11. LIU J., LISSENS W., SILBER S.J., DEVROEY P., LIBAERTS I., VAN STEIRTHEGEM A. : Birth after preimplantation diagnosis of the cystic fibrosis $\triangle F 508$ mutation by polymerase chain reaction. J. Am. Med. Ass., 1994, 272 : 1858-1860.

12. MESCHEDE D., LEMCKE B., EXELER J.R. et al. : Chromosome abnormalities in 447 couples undergoing intracytoplasmic sperm injection - prevalence, types, sex distribution and reproductive relevance. Hum. Reprod., 1998, 13 : 576-582.

13. RAY P., GIGAREL N., BONNEFONT J.P. et al. : First specific PGD for ornithine transcarbanylase deficiency. Pren. Diagn., 2000, $20: 1048-1054$.

14. RAY P.F., MUNNICH A., NISAND I., FRYDMAN R., VEKEMANS M., VIVILLE S. : The place of "social sexing" in medicine and science. Hum. Reprod., 2002, 17 : 248-249.

15. SCRIVEN P.N., HANDYSIDE A.H., OGILVIE C.M. : Chromosome translocations: segregation modes and strategies for preimplantation genetic diagnosis. Prenat. Diagn., 1998, 18 : 1437-1449.

16. STROM C.M., STROM S., LEVINE E., GINSBERG N., BARTON J., VERLINSKY Y. : Obstetric outcomes in 102 pregnancies after preimplantation genetic diagnosis. Am. J. Obstet. Gynecol., 2000, $182: 1629-1632$.

17. TARIN J.J., HANDYSIDE A.H. : Embryo biopsy strategies for preimplantation diagnosis. Fertil. Steril., 1993, 59 : 943-952.

18. VAN DER VEN K., PESCHKA B., MONTAG M., LANGE R., SCHWANITZ G., VAN DER VEN H.H. : Increased frequency of congenital chromosomal aberrations in female partners of couples undergoing intracytoplasmic sperm injection. Hum. Reprod., 1998, $13: 48-54$.

19. WILTON L., WILLIAMSON R., McBAIN J., VOULLAIRE L.: Use of comparative genomic hybridization to karyotype embryos prior to implantation : the achievement of a successful pregnancy. Hum. Reprod., 2001, 16 : abstract book 1, 33.

\section{ABSTRACT}

Preimplantation Genetic Diagnosis: update

Nelly ACHOUR-FRYDMAN, Gérard TACHDJIAN, Pierre RAY, Serge ROMANA, Samir HAMAMAH, Sabine MARCADET-FREDET, Violaine KERBRAT, Renato FANCHIN, Jacques KADOCH, Tania ATTIE, M. LELORC'H, Michel VEKEMANS, Arnold MUNNICH, René FRYDMAN

This paper reports the birth of the first fourteen infants conceived after preimplantation genetic diagnosis (PGD) in our unit. Fifty-nine couples were enrolled between January 2000 and July 2001. They had a total of 71 oocyte pick-up cycles. The collected oocytes were inseminated by intracytoplasmic sperm injection. The resulting embryos were biopsied on the third day of development and genetic analysis was performed on the same day. Most of the embryo transfers were carried out on the fourth day. The 71 oocyte pick-up cycles yielded 872 oocytes of which 731 were suitable for intacytoplasmic sperm injection. Among the 505 embryos obtained, 421 embryos were biopsied and genetic diagnosis was performed for $312(74 \%)$ of them. 127 embryos were transferred during $\mathbf{5 8}$ transfer procedures. There were 18 biochemical and 12 ongoing (7 singles, 4 twins and 1 triple) pregnancies. Sixteen infants have been born and 2 are expected. PGD now constitutes an alternative for couples at risk of transmission of a serious and incurable genetic disease.

Key words: Preimplantation genetic diagnosis, chromosomal abnormality, single gene disease 